EPJ Web of Conferences 103, 01004 (2015)

DOI: 10.1051/epjconf/201510301004

(C) Owned by the authors, published by EDP Sciences, 2015

\title{
Electromagnetic Field Scattering on rf-SQUID Based Metasurfaces
}

\author{
J.G Caputo ${ }^{1}$, I.R. Gabitov ${ }^{2,3 a}$, Zh. Kudyshev ${ }^{3}$, T. Kupaev ${ }^{4,5}$, and A.I. Maimistov ${ }^{6,7}$ \\ ${ }^{1}$ Laboratoire de Mathématiques de I'INSA de Rouen, Av. de I'Universite, Saint-Etienne du Rouvray, 76801 France \\ ${ }^{2}$ Department of Mathematics, University of Arizona, Tucson, AZ, 85704, USA \\ ${ }^{3}$ Skolkovo Institute of Science and Technology, Skolkovo Innovation Center, Building 3, Moscow 143026, Russia \\ ${ }^{4}$ Department of Applied and Computational Mathematics and Statistics, 153 Hurley Hall, Notre Dame, IN 46556 \\ ${ }^{5}$ Department of Molecular Physics, National Research Nuclear University, Moscow Engineering Physics Institute, \\ Kashirskoe sh. 31, Moscow, 115409 Russia \\ ${ }^{6}$ Department of Solid State Physics and Nanostructures, National Research Nuclear University, Moscow \\ Engineering Physics Institute, Kashirskoe sh. 31, Moscow, 115409 Russia \\ ${ }^{7}$ Department of General and Applied Physics, Moscow Institute for Physics and Technology, Institutskii lane 9 , \\ Dolgoprudny, Moscow region, 141700 Russia
}

\begin{abstract}
Electromagnetic field scattering on a 2D array of rf-SQUIDs is considered. We show that the scattering changes for large amplitudes of the incident electromagnetic wave; above a critical amplitude, two different refraction states occur (bistability). In particular, for these two states, the transmitted wave polarization and angle of refraction are different. One could then switch the direction of propagation of the electromagnetic wave and its polarization with a "thin film", whose thickness is much smaller than the wavelength.
\end{abstract}

The concept of metasurfaces introduced in the early 2000 [1] became quite popular after the demonstration of generalized refraction of an electromagnetic field $[2,3]$ by a 2D array of plasmonic structures. The interest in this subject is motivated by potentials for developing sub-wavelength size devices capable to control light, which could be a step towards high-integration optical circuits. Taking into account the tremendous impact of nonlinear optics on light-based technology it is of considerable importance to study the nonlinear properties of metasurfaces.

The radio frequency superconducting quantum interference device (rf-SQUID) which was invented in 1964 [4] is a natural building block for a nonlinear metasurface. It is a superconducting split ring with a Josephson junction mounted in the gap. We consider a 2D array of such rf-SQUIDs where each rf-SQUID has the same orientation and inclination angle $\theta$ with respect to the plane of the array. The linear scattering of the normally incident plane electromagnetic wave on such an array for a weak incident field was studied in [5]. It was shown that the reflected wave experiences a rotation of the polarization (analog of Kerr's effect); this reflected wave is oriented along the normal to a split ring so its direction depends only on the angle of inclination of the rf-SQUIDs. The angle of reflection and the polarization of the transmitted wave depend both on the angle of inclination and on the carrier frequency. To analyze the weakly nonlinear case following [5] we consider the system of dimensionless equations governing the interaction of the magnetic component of the electromagnetic field with the thin film containing the rf-SQUIDS

\footnotetext{
${ }^{a}$ Corresponding author: ildar.gabitov@gmail.com
} 


$$
\dddot{\varphi}+\gamma \ddot{\varphi}+\varphi+\beta \sin \varphi=f, f=-\alpha(\dot{\varphi}+\dot{f})+f_{\text {in }} .
$$

Here $\varphi, \gamma$ are the phase difference and the losses of the Josephson junction while $\beta$ is proportional to a bios current in the rf-SQUID. The Thompson frequency $\omega_{T}^{2}=(L C)^{-1}$ of the $L C$ - circuit (inductance and capacity) representing the split ring is rescaled to unity. The function $f=\vec{h} \cdot \vec{n}$ is the scalar product of the dimensionless magnetic component of the electromagnetic field and the normal to the split ring, the term $f_{\text {in }}$ is a similar scalar product for the incident field, and the coefficient $\alpha$ describes losses due to radiation. It should be noted that $\vec{h}$ corresponds to the transmitted field. We take function $f_{\text {in }}$ in the form of $f_{\text {in }}=k \cos \omega t$ and expand $\sin \varphi \approx \varphi-\varphi^{2} / 6 \sin \varphi$. After straightforward algebraic manipulations, (see [6]) we obtain the critical threshold of $k$ for the bifurcation of stationary solutions:

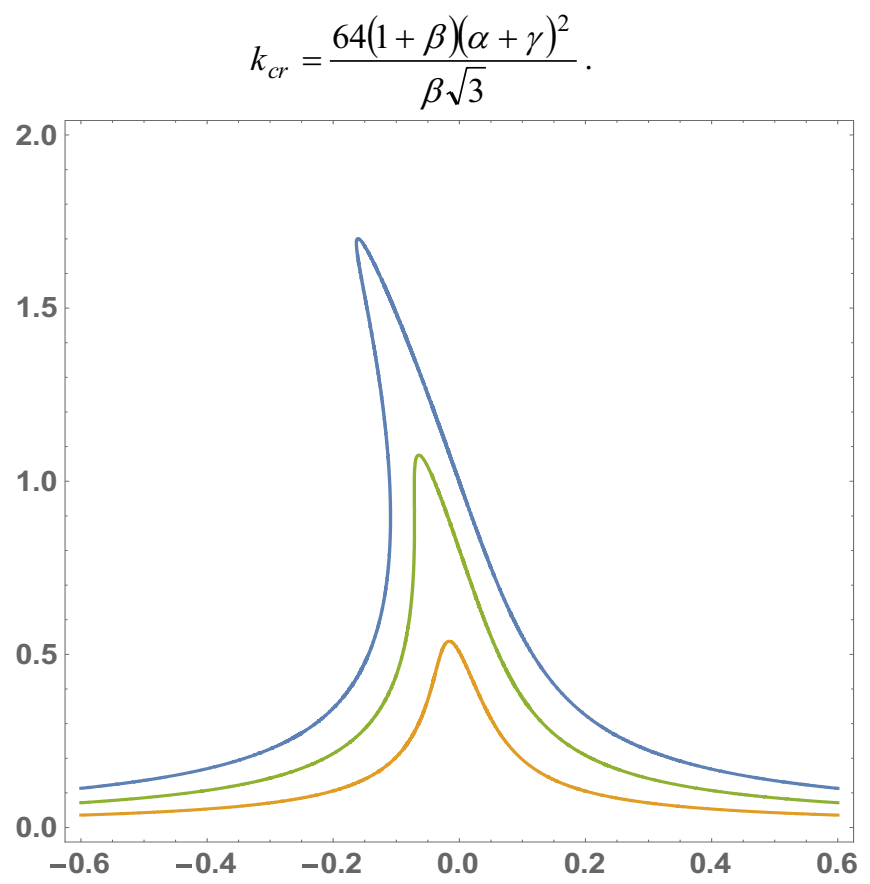

Figure 1. Figure shows dependence of the phase $\varphi$ on the detuning $\varepsilon$ of the carrier frequency from the resonance frequency. The line in amber is the linear response of the rf-SQUIDs, the green line is for a critical value of the incident field and the blue line is for the supercritical regime where there is bistability.

The dependence of the phase $\varphi=\varphi(\varepsilon, k)$ on the frequency detuning $\varepsilon=\omega-\omega_{T}$ for different values of $k$ and a fixed value of the inclination angle is illustrated on Fig. 1. Amber, green, and blue colors correspond to a linear, critical and bistable cases respectively. The bistable regime, in accordance to equations (1), means the existence of two scattering configurations with different angles of refraction and polarizations. The transition from one state to another can be achieved by tuning the frequency of the incident field.

In conclusion, we generalized results of the paper [5] for the case of a nonlinear response of the rfSQUDs array to the incident electromagnetic field. If the amplitude of the incident field exceeds a critical value (2), then there are two stable states of field refraction from the array of rf-SQUDs. Switching from one state to another is controlled by the detuning of the carrier frequency from the resonance frequency of the rf-SQUIDs.

Acknowledgments: The research of A.I.M. was supported by the Russian Science Foundation (Project No. 14-22-00098). 


\section{References}

1. Ze'ev Bomzon, Vladimir Kleiner, and Erez Hasman, Opt. Lett., 26 (18), pp. 1424-1426, (2001)

2. N. Yu, P. Genevet, M.A. Kats, F. Aieta, J.P. Tetienne, F. Capasso, Z. Gaburro, Science. 334 (6054):333-7, (2011)

3. Ni. Xingjie, K. Naresh, A. Emani, V. Kildishev, A. Boltasseva, V. M. Shalaev, , Science 335 (6067) p. 427 (2012)

4. R. C. Jaklevic, J. Lambe, A. H. Silver, and J. E. Mercereau, Phys. Rev. Letters 12 (7): 159-160, (1964)

5. J.-G. Caputo, I. Gabitov, and A. I. Maimistov, Phys. Rev. B 91, 115430 (2015)

6. L.D. Landau, E.M. Lifshitz, Mechanics. Vol. 1 (3rd ed., Butterworth-Heinemann, ISBN 978-07506-2896-9, 1976) 